\title{
Herramientas Software para Enseñanzas Relacionadas con la Energía Solar Fotovoltaica
}

\author{
Rafael Peña Capilla ${ }^{a}$, María del Pilar García Díaz ${ }^{a}$, Cristina Alén Corderoa ${ }^{a}$ Pablo \\ Díaz Villar ${ }^{a}$, Ricardo Mallol Poyato ${ }^{a}$, Ana M. Díez-Pascual ${ }^{b}$.
}

a'Departamento de Teoría de la Señal y Comunicaciones (rafael.pena@uah.es, pilar.garcia@uah.es, cristina.alen@uah.es, pablo.diaz@uah.es, ricardo.mallol@uah.es), ${ }^{b}$ Departamento de Química Analítica, Química Física e Ingeniería Química (am.diez@uah.es).

\begin{abstract}
This paper describes different simulation tools developed in the Universidad de Alcalá in order to teach photovoltaic energy engineering. A web application is compared to other tools, such as spreadsheets and applications in Matlab environment, developed by the authors.
\end{abstract} In all the cases, the tools are focused on learning project engineering.

Keywords: E-learning, Solar Engineering, Software Tools.

\section{Resumen}

En este trabajo, se describen diferentes herramientas de simulación empleadas en asignaturas de la Universidad de Alcalá para la enseñanza de la energía solar fotovoltaica.

Se compara el uso de una aplicación web con otras herramientas como hojas de cálculo y aplicaciones en entorno Matlab. Todas las herramientas descritas han sido desarrolladas por los autores, haciendo especial énfasis en su enfoque didáctico, sin por ello perder todo el rigor y la funcionalidad necesarios en este tipo de simuladores.

Palabras clave: E-learning, Energías Renovables, Herramientas software.

\section{Introducción}

Las herramientas software son comúnmente empleadas como apoyo al desarrollo de proyectos de ingeniería en muy diferentes campos. En particular, en el área de las energías renovables (solar fotovoltaica, térmica, eólica...), tecnologías en fuerte expansión a lo largo de los últimos años.

En el campo de la energía solar, existen programas comerciales que permiten dimensionar las instalaciones y evaluar su rendimiento. Algunos de los ejemplos más representativos son PVGIS, PVSYS o Censol (Dunlop y Wald, 2006). 
Además, es muy común emplear hojas de cálculo o programas desarrollados en lenguajes como Matlab, Python, Octave, etc.

Dichas aplicaciones suelen estar enfocadas a la fase de diseño de la instalación, en la que ya se conocen con cierto detalle las características básicas de ésta: emplazamiento de los componentes, características constructivas de dicho emplazamiento, trazado aproximado del cableado de la instalación, etc. Además, las mencionadas herramientas están concebidas para ser utilizadas por proyectistas ya formados, con los conocimientos teórico-prácticos adquiridos y con cierta experiencia en el sector.

En consecuencia, todas estas herramientas suelen ser poco eficientes a la hora de realizar tareas propias de la fase de anteproyecto, en la que es fundamental obtener un dimensionado aproximado de la instalación en poco tiempo y con suficiente precisión. Y teniendo en cuenta que, en ocasiones, las citadas herramientas son empleadas por ingenieros en formación.

Además, los simuladores para instalaciones solares no están diseñados con un enfoque didáctico, que permita reforzar los conceptos teóricos que subyacen tras las ecuaciones empleadas.

\section{Objetivos}

El objetivo principal del trabajo es analizar las diferentes opciones disponibles como herramientas de diseño de proyectos de energía solar enfocadas al aprendizaje de la ingeniería. E identificar las principales ventajas e inconvenientes de cada alternativa en cuanto a su eficacia en los aspectos didácticos.

Se presenta en primer lugar el proyecto PVLEARNING, desarrollado en un entorno web. La herramienta asiste al ingeniero en la realización de diversas tareas de diseño de sistemas fotovoltaicos entre las que cabe destacar las siguientes:

- Evaluación de la radiación solar disponible en el emplazamiento seleccionado.

- Selección de los módulos fotovoltaicos y del inversor o inversores.

- Configuración del campo solar (filas de módulos en serie y/paralelo) y del inversor.

- Cálculo del rendimiento de la instalación

También se han empleado con el mismo propósito una hoja de cálculo desarrollada por los autores y un programa en entorno Matlab. En el artículo se discuten las ventajas e inconvenientes de las diferentes opciones, y su influencia en los procesos de enseñanza / aprendizaje. 
Rafael Peña Capilla, María del Pilar García Díaz, Cristina Alén Cordero, Pablo Díaz Villar, Ricardo

Mallol Poyato, Ana M. Díez-Pascual

\section{Desarrollo de la innovación}

Se describen a continuación las herramientas desarrolladas en la Universidad de Alcalá para la simulación de sistemas solares fotovoltaicos.

\subsection{Entorno web PVLEARNING}

El proyecto PVLEARNING se ha desarrollado en sucesivas fases, que se describen a continuación.

- Elaboración de un modelo teórico para la producción energética de instalaciones fotovoltaicas con conexión a red.

Se ha realizado una búsqueda bibliográfica para determinar qué modelos teóricos se han utilizado hasta la fecha como base de los actuales programas de simulación. En particular, se han seleccionado los descritos en (Schmid 1992; Lorenzo 2002, Lorenzo 2006, Caamaño \& Egido, Alonso \& Chenlo).

Con esta base, se ha desarrollado un nuevo modelo con las características ya descritas: enfocado al aprendizaje de la ingeniería de proyectos pero manteniendo toda la funcionalidad y precisión en su utilización en la fase de diseño de proyecto.

Así, el modelo de cálculo empleado toma como base fundamentos teóricos que cumplen un compromiso entre sencillez y rigurosidad. Por ello, emplea pocos parámetros que, además, son de fácil obtención e interpretación.

PVLEARNING calcula la energía generada por un sistema fotovoltaico mediante la siguiente expresión:

$$
E_{A C}=P \cdot G_{a e f} \cdot F P
$$

Siendo P la potencia de pico del generador fotovoltaico, Gaef el valor de la irradiación anual efectiva en el plano de los módulos (en horas de sol de pico) y FP un factor que engloba las pérdidas del sistema.

Para el cálculo de dicho factor, se han tenido en cuenta las siguientes pérdidas:

- Pérdidas debidas al rendimiento del inversor

- Pérdidas debidas a la presencia de suciedad en los módulos

- Pérdidas debidas a la temperatura

- Pérdidas por sombreado

- Pérdidas espectrales y angulares

- Pérdidas por dispersión de parámetros (mismatch)

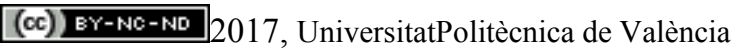


- Pérdidas por efecto Joule en el cableado de corriente continua y corriente alterna

- Otras pérdidas, como la disminución del rendimiento de los componentes a baja irradiancia o la no disponibilidad de éstos en paradas por mantenimiento o por averías.

El detalle de las ecuaciones utilizadas para cada una de los factores de pérdidas queda fuera del alcance de este artículo.

PVLEARNING 10-11

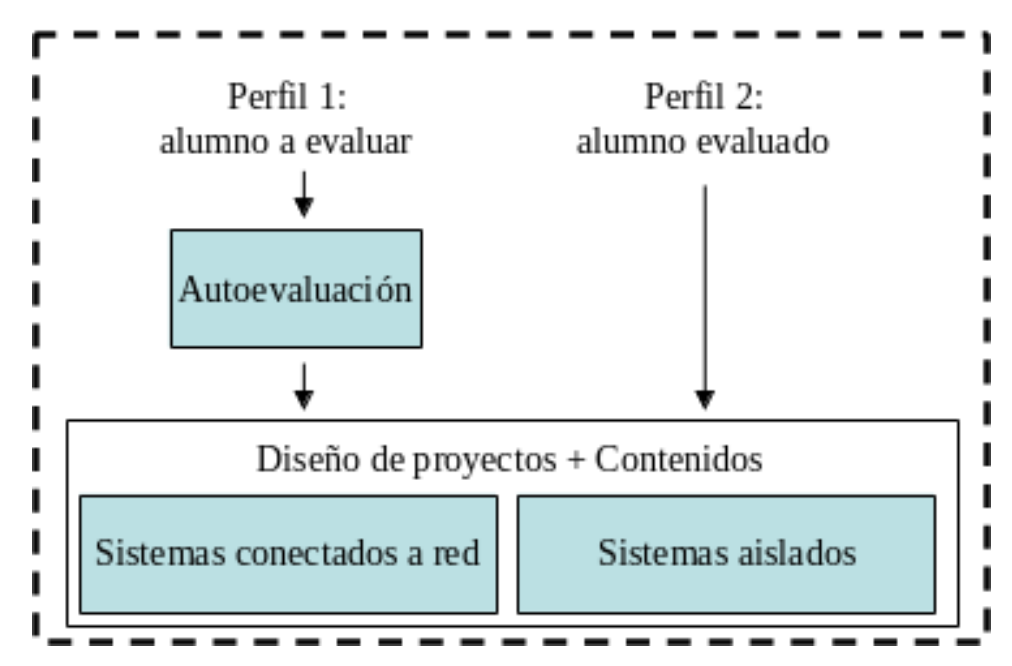

Figura 1: esquema simplificado de la estructura de la plataforma PVLEARNING.

- Desarrollo del programa fuente para la simulación de sistemas fotovoltaicos. Verificación de los resultados.

Tras obtener las expresiones analíticas del modelo, se ha realizado la herramienta software que constituye el núcleo de cálculo de PVLEARNING. Para ello, se ha utilizado Matlab, lenguaje de programación muy adecuado para este tipo de simulaciones.

El programa se ha probado exhaustivamente, realizando múltiples simulaciones de sistemas fotovoltaicos. En particular, se ha comparado con PVSYST, la herramienta más utilizada en este campo.

Se han obtenido diferencias entre los resultados obtenidos con PVLEARNING y los de PVSYST menores del $2 \%$ en sistemas fijos y menores del $5 \%$ en sistemas con seguimiento. Estos valores son muy notables, teniendo en cuenta la sencillez del modelo desarrollado y la facilidad de uso de la aplicación.

- Desarrollo de la interfaz gráfica para la utilización del software en un entorno web.

Tras obtener el núcleo del programa y una vez depurado éste, se ha implementado la interfaz gráfica para su utilización en Web. Dicha interfaz ha sido realizada con

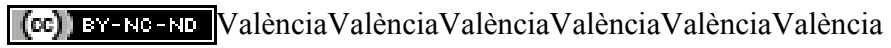


los lenguajes PHP y Java Script. Para las bases de datos empleadas por la aplicación se ha utilizado MySQL.

- $\quad$ Puesta en marcha de la plataforma desarrollada. Aplicación a la mejora de la docencia en asignaturas del Área de Ingeniería Industrial de la Universidad de Alcalá.

Los usuarios de PVLEARNING pueden acceder, previa autenticación, a dos perfiles o modalidades diferentes (véase figura 1).

- Modalidad de autoevaluación

Los usuarios que acceden al tipo de perfil 1, pueden crear un proyecto con toda la funcionalidad del sistema. Sin embargo, la solución para el problema seleccionado no está disponible hasta que éste ha introducido la suya propia, tras haberla calculado por su cuenta. En ese momento, el sistema proporciona la solución correcta y la compara con la introducida previamente. En el caso de que la comparación sea favorable (solución correcta), el usuario pasa a tener perfil 2 , de diseño de proyecto.

- Modalidad de diseño de proyecto

Los usuarios que acceden con este tipo de perfil o bien ya han adquirido los conocimientos básicos, asistidos por la herramienta en la modalidad de autoevaluación, o están autorizados directamente por los administradores. Esta última opción permite que ingenieros ya formados no tengan que pasar por el filtro del perfil 1.

La Figura 2 muestra la interfaz principal en ésta modalidad, para el caso de instalaciones conectadas a la red, en la que el programa funciona como un simulador "al uso". Dicha interfaz dispone de dos bases de datos, una para la selección del emplazamiento de la instalación (primer paso a la hora de diseñar un sistema) y otra para la selección de los principales componentes (paneles solares e inversor). 




Al elegir una determinada ubicación, la interfaz asigna los valores correspondientes para la irradiación disponible en ella. El usuario también debe elegir el tipo de instalación (fija, con seguimiento en un eje o en dos ejes). En el primer caso, además habrá de introducir la orientación o azimut de los paneles y su inclinación con respecto a la horizontal. Todos estos parámetros son utilizados por el modelo descrito anteriormente.

También es necesario seleccionar el grado de suciedad de los módulos, sin suciedad en el caso de que estén limpios y grado medio para el caso de que tengan el nivel de suciedad típico esperado en instalaciones solares.

Por último, el usuario puede modificar los factores de pérdidas calculados por el programa, o bien dejar los valores por defecto (opción recomendada).

Una vez configurada la simulación, el programa muestra los resultados que aparecen en la figura 3: la energía anual producida por el sistema, la productividad final, el performance ratio (PR) y los valores de irradiación anual característicos del sistema (con y sin sombras).

Además de sistemas conectados a la red eléctrica, PVLEARNING permite dimensionar sistemas fotovoltaicos aislados (figura 4). El modelo empleado para este tipo de sistemas es una simplificación del método del mes peor, de amplia utilización. Los parámetros de entrada para dicho modelo son la irradiación mensual en el plano del generador en la ubicación seleccionada, el consumo anual previsto y las características básicas de los paneles solares y de las baterías a emplear.

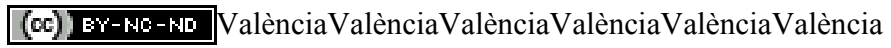


Rafael Peña Capilla, María del Pilar García Díaz, Cristina Alén Cordero, Pablo Díaz Villar, Ricardo Mallol Poyato, Ana M. Díez-Pascual

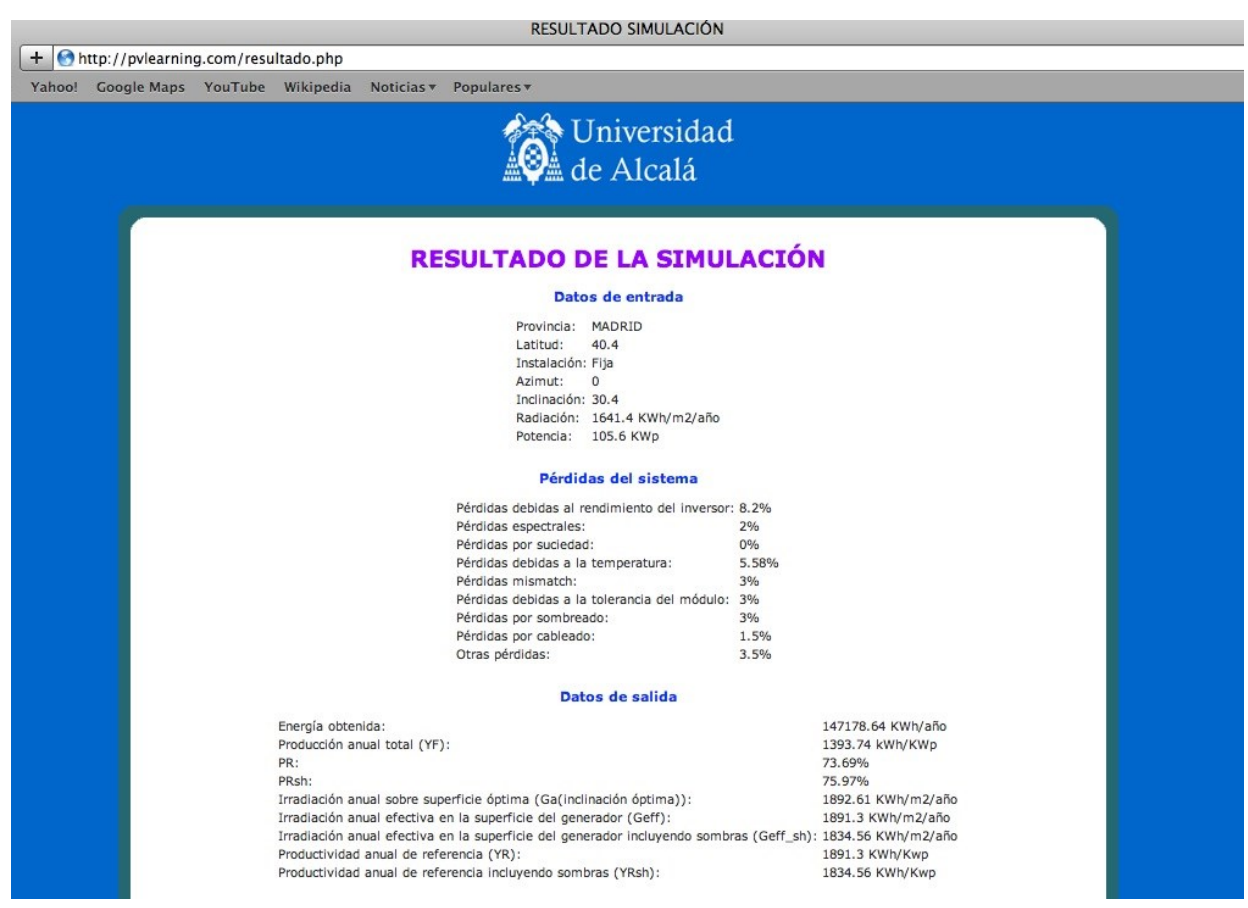

Figura 3: formulario de salida de PVLEARNING con los resultados para un sistema conectado a la red

\begin{tabular}{|c|c|c|c|c|c|c|c|c|c|}
\hline \multicolumn{10}{|c|}{ hittp://pvlearning.com/formulario1.php } \\
\hline \multicolumn{10}{|c|}{ o! Google Maps YouTube Wikipedia Noticiasv Populares- } \\
\hline \multicolumn{10}{|c|}{ SIMULACIÓN DE SISTEMAS FOTOVOLTAICOS AISLADOS DE LA RED } \\
\hline \multicolumn{6}{|c|}{ UBICACIÓN } & \multicolumn{4}{|c|}{ COMPONENTES } \\
\hline \multirow{3}{*}{\multicolumn{2}{|c|}{ |usicación: }} & \multirow{3}{*}{\multicolumn{2}{|c|}{  }} & & & Móoulo: & Genérico & \multicolumn{2}{|l|}{$=0$} \\
\hline & & & & & & \multirow{5}{*}{ 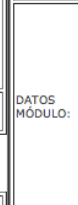 } & Nombre & \multicolumn{2}{|l|}{ Genérico } \\
\hline & & & & er datos por pro & & & Potencia máx (WP) & \multicolumn{2}{|l|}{75} \\
\hline \multicolumn{2}{|c|}{ inclinación (o): } & \multicolumn{4}{|c|}{$60 \div$} & & Tensión pmp ( (v) & \multicolumn{2}{|l|}{17.3} \\
\hline \multirow{2}{*}{\multicolumn{6}{|c|}{ CONSUMOS DC }} & & $\begin{array}{l}\text { Corriente pmp (A) } \\
\text { Tensión de circutoto abierto }\end{array}$ & \multicolumn{2}{|l|}{4.34} \\
\hline & & & & & & & $\mid \begin{array}{l}\text { Moriente de cotocircuito } \\
\text { Cons }\end{array}$ & \\
\hline TIPo & Número & Potencia(w) & Hores/dia & $\begin{array}{l}\text { Consumom } \\
\text { diero(W.h) } \\
\end{array}$ & $\begin{array}{l}\text { Consumo } \\
\text { diario(A.h) } \\
\end{array}$ & Bateria: & & & \\
\hline Televisión & 0 & 100 & 2 & 0 & 0.00 & \multirow{2}{*}{ 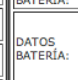 } & Cappaidad C100 (Ah) & \multicolumn{2}{|l|}{250} \\
\hline Firigoritico & 0 & 100 & 2 & 0 & 0.00 & & Tension (w) & \multicolumn{2}{|l|}{12} \\
\hline 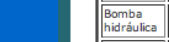 & 0 & 150 & $\mid 1$ & 0 & 0.00 & \multicolumn{4}{|c|}{ PARÁmetros DEL SISTEMA } \\
\hline Bonbillas & 0 & 11 & 4 & 0 & 0.00 & \multirow{2}{*}{\multicolumn{2}{|c|}{ Factor de seguridad del generador }} & & \\
\hline Ordenasort & 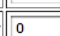 & $\longdiv { 2 0 0 }$ & $\longdiv { 2 }$ & 0 & 0 & & & & 1.25 \\
\hline otros & $\sqrt{0}$ & $\longdiv { 1 0 0 }$ & $\sqrt{1}$ & 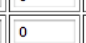 & 0.00 & \multicolumn{2}{|c|}{ Dias de autonomia de la bateria } & 5 & \\
\hline & TOTAL & 0 & & 0 & 0.00 & \multicolumn{2}{|l|}{ POmax } & & 0.8 \\
\hline & & & & & & \multicolumn{2}{|c|}{ Rendimiento del inversor } & & 0.9 \\
\hline Calcular & consum & & & & & \multicolumn{2}{|c|}{ Tensín del sistema (V) } & 1 & \\
\hline & \multicolumn{5}{|c|}{ CONSUMOS AC } & & & & \\
\hline Tripo & Número & Potencia(W) & Horess/dia & 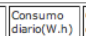 & $\begin{array}{ll}\begin{array}{c}\text { Consumon } \\
\text { diaribitAn }\end{array} \\
\end{array}$ & & & & \\
\hline Trevisision & 0 & 100 & $\mid \sqrt{2}$ & 0 & 0.00 & & & & \\
\hline \begin{tabular}{|l|l} 
Figigoritico & \\
\end{tabular} & 0 & 100 & 2 & 0 & 0.00 & & & & \\
\hline 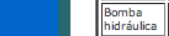 & 0 & 150 & 1 & 0 & 0.00 & & & & \\
\hline
\end{tabular}

Figura 4: formulario de entrada de datos de www.pvlearning.com correspondiente a la simulación de sistemas aislados.

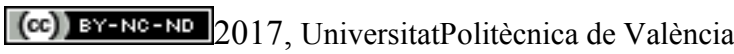
Congreso In-Red (2017) 
Tras estimar los consumos de la instalación y seleccionar los componentes y la ubicación, el programa calcula la potencia necesaria para los paneles y la capacidad de las baterías del sistema.

\subsection{Herramientas de simulación en forma de hoja de cálculo y en entorno Matlab}

El modelo teórico para la simulación de instalaciones fotovoltaicas se ha programado en Matlab, lenguaje de programación muy adecuado para este tipo de simulaciones, y también se ha desarrollado una hoja de cálculo con las mismas ecuaciones (figuras 5 y 6). En ambos casos, se han incluido las herramientas para simular instalaciones conectadas y aisladas de la red.

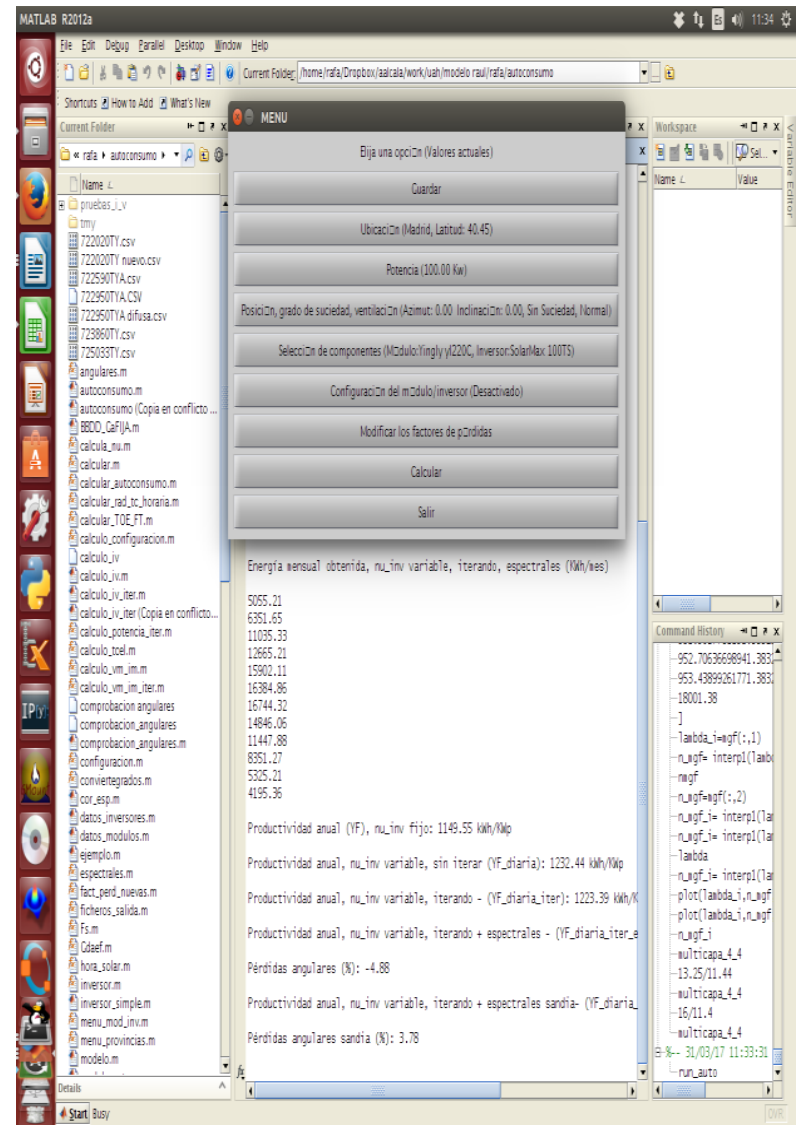

Figura 5: herramienta desarrollada en entorno Matlab para la simulación de sistemas fotovoltaicos conectados a la red. 


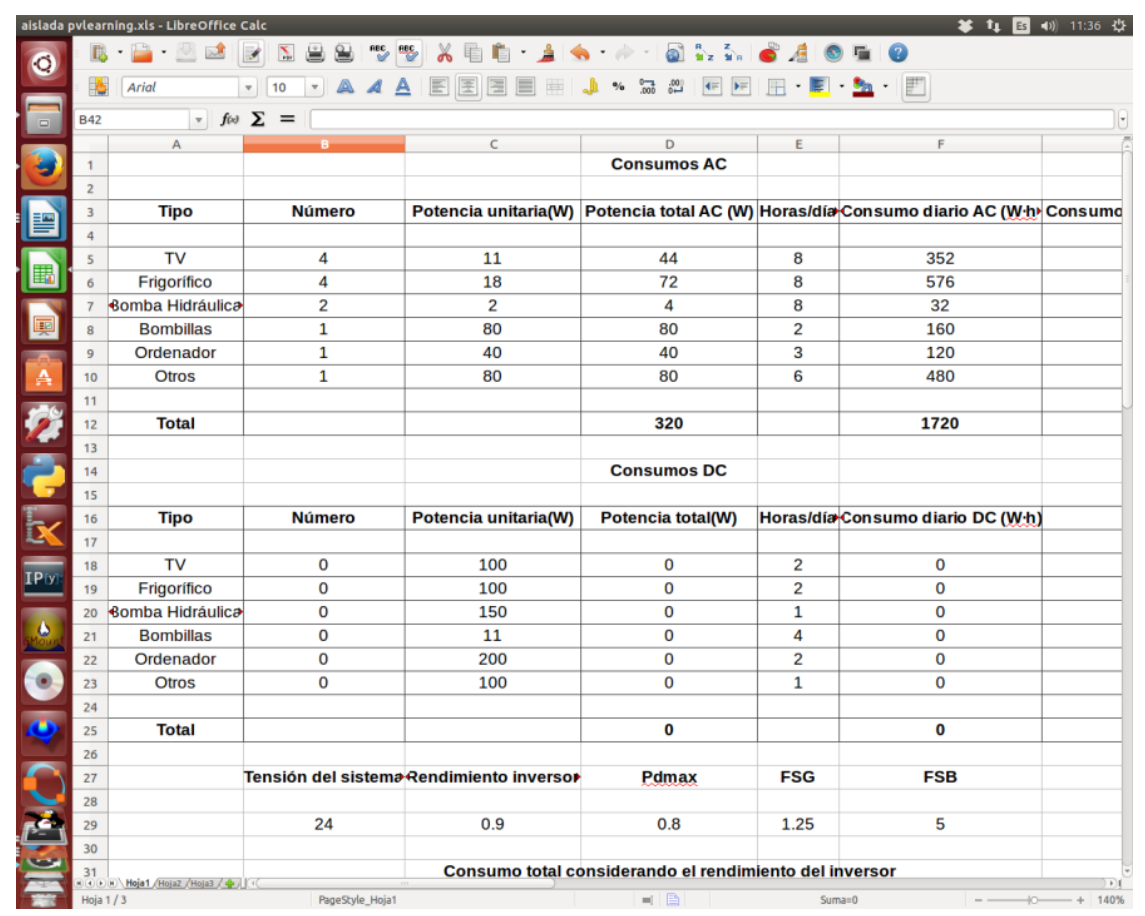

Figura 6: hoja de cálculo desarrollada para la simulación de sistemas fotovoltaicos aislados de la red.

\section{Resultados}

La primera opción presentada, la web PVLEARNING, ha sido utilizada en asignaturas de grado y postgrado del Área de Ingeniería Industrial de la Universidad de Alcalá. Además, el programa servirá también para la enseñanza de la energía solar en cursos de formación impartidos a profesionales.

En particular, desde el curso 2010-2011 hasta el 2014-2015, PVLEARNING se empleó en las asignaturas Suministro Eléctrico de Instalaciones Autónomas mediante Energías Renovables, del Máster Universitario en Tecnologías de la Información y las Comunicaciones (optativa de primer curso) y Proyecto Fin de Carrera, de la Ingeniería Técnica Industrial, especialidad en Electrónica Industrial (troncal de tercer curso, recientemente extinguida). Además, se está utilizando en asignaturas optativas del Grado en Ingeniería en Electrónica y Automática Industrial y en trabajos fin de grado y fin de máster realizados en el área de Ingeniería Eléctrica.

Como se comentó con anterioridad, una de las características diferenciadoras del proyecto PVLEARNING es su enfoque al aprendizaje de la ingeniería solar fotovoltaica. Las simulaciones se emplean en el aula como complemento a las clases teóricas, al final de éstas, para servir de nexo entre los contenidos puramente teórico-académicos y los relacionados con las tareas de diseño de proyecto-ingeniería. De esta manera, se refuerzan los conceptos expuestos y se hace énfasis en su utilidad práctica. Además, el alumno toma 
el primer contacto con una herramienta de diseño como las que habrá de utilizar en su trayectoria profesional. Todo ello no sólo tiene como consecuencia un aumento de la eficacia del proceso enseñanza - aprendizaje, si no que también redunda en una mayor motivación del alumno a la hora de estudiar conceptos con un elevado nivel de dificultad.

Además, los estudiantes emplean PVLEARNING para realizar trabajos de evaluación de la asignatura, de manera autónoma (de manera semejante a como lo hacen los estudiantes de Trabajo fin de Grado). Para ello, han de superar la fase de autoevaluación, descrita con anterioridad.

Con respecto a la influencia de la herramienta en el proceso enseñanza-aprendizaje, ésta se ha utilizado sobre una muestra de 90 alumnos, en las asignaturas mencionadas con anterioridad. La propia página web ha servido como herramienta para la recogida de la información, puesto que los estudiantes hubieron de acceder a la modalidad de autoevaluación antes de poder realizar las simulaciones correspondientes a su trabajo.

Los resultados obtenidos son muy positivos. Con respecto a la experiencia anterior con las mismas asignaturas, se ha detectado un menor número de abandonos y una mejora significativa en la calidad de los trabajos de evaluación presentados. Cabe achacar esta mejora a los siguientes factores relacionados con la utilización de PVLEARNING:

- La herramienta sirve de nexo entre los contenidos puramente teórico-académicos y los relacionados con las tareas de diseño de proyecto-ingeniería. Así, la utilización de las TIC en el estudio permite reforzar los conceptos, haciendo énfasis en su utilidad práctica.

- Hay un aumento en la motivación del alumno a la hora de estudiar conceptos con un elevado nivel de dificultad. Esto es consecuencia del empleo de una herramienta de diseño como las que habrá de utilizar en su trayectoria profesional.

- Se ha detectado un mayor nivel de participación en clase, como consecuencia del aumento de la motivación de los estudiantes.

Con respecto a PVLEARNING, el programa en Matlab y la hoja de cálculo anteriormente descritos presentan las siguientes desventajas:

- Requieren de la instalación previa en el ordenador del alumno, que además debe tener un sistema operativo compatible con Matlab o con Microsoft Office / LibreOffice / OpenOffice.

- La implementación de la modalidad de autoevaluación es posible, pero el envío y registro de los resultados no es automático, debe ser realizado de manera "manual" por los estudiantes y el profesor. En el caso de PVLEARNING, la propia página web sirve como herramienta para la recogida de la información

- La incorporación de los conceptos teóricos que hay tras las ecuaciones del modelo se puede realizar tanto en entorno Matlab como en una hoja de cálculo. Sin embargo, el entorno web permite una integración visual mucho más atractiva, más intuitiva, y una mayor usabilidad. 
Rafael Peña Capilla, María del Pilar García Díaz, Cristina Alén Cordero, Pablo Díaz Villar, Ricardo Mallol Poyato, Ana M. Díez-Pascual

- Ambas opciones no permiten la interacción directa entre el estudiante y el profesor, algo que si permite la alternativa "online".

\section{Conclusiones}

En este trabajo, se presentan 3 herramientas para el diseño de proyectos de energía solar, todas ellas enfocadas al aprendizaje de la ingeniería; y se identifican las principales ventajas e inconvenientes de cada alternativa en cuanto a su eficacia en los aspectos didácticos.

Así, se presentan el proyecto PVLEARNING, desarrollado en un entorno web, una hoja de cálculo y un programa en entorno Matlab, todos desarrollados por los autores.

En todos los casos, las herramientas sirven de nexo entre los contenidos puramente teóricoacadémicos y los relacionados con las tareas de diseño de proyecto, reforzando los conceptos y haciendo énfasis en su utilidad práctica. Se ha observado un aumento en la motivación del alumno, como consecuencia del empleo de una herramienta de diseño como las que habrá de utilizar en su trayectoria profesional; y se ha detectado un mayor nivel de participación en clase.

Además de aspectos de índole más práctico (no es necesaria la instalación previa en el ordenador del alumno, ni disponer de un sistema operativo compatible), PVLEARNING permite integrar herramientas de autoevaluación, de recogida de datos relacionados con la evolución del aprendizaje y la integración de contenidos teóricos de forma mucho más atractiva y más intuitiva; y en general, una mayor usabilidad.

\section{Referencias}

ALONO, M., CHENLO, F. Estimación de la energía generada por un sistema fotovoltaico conectado a red. $\quad<$ http://www.energiasrenovables.ciemat.es/adjuntos_documentos/Alonso-chenlo.pdf $>$ [Consulta: mayo 2016].

DUNLOP, E., WALD, L. (2006). Solar Energy Resource Management for Electricity Generation from Local to Global Scale. New York: Nova Science Publishers.

LORENZO, E. (2006). Radiación Solar y Dispositivos Fotovoltaicos. Volumen II. Sevilla: Editorial Progensa.

LORENZO, E. (2002). La Energía que Producen los Sistemas Fotovoltaicos Conectados a la Red. Era Solar, 107, 22-28.

SCHMID, J. (1992). PV in Buildings. Actas del XI Congreso europeo de Energía Solar fotovoltaica. 1659-1663. 